\title{
Study of Optical Property of Gel Grown Mercuric Iodate Crystals
}

\author{
Amit B Patil \\ P.G. Department of Physics, Pratap College, Amalner - 425401, (MAH) India
}

\begin{abstract}
Mercuric Iodate Crystals were grown by a simple gel technique using diffusion method. The optimum growth conditions were established for the growth of these crystals by changing various parameters such as $p H$ of the gel solution, gel concentration, gel setting time, concentrations of reactants etc. The grown Mercuric Iodate crystals were spherical in shape. These crystals were opaque. The crystals were characterized using UVVIS Specrophotometrry.
\end{abstract}

.Keywords. Gel technique, mercuric iodate crystals, UV-VIS Specrophotometrry.

\section{Introduction:}

A variety of crystals required for the purpose of research and application can be grown in silica gels. The gel medium prevents turbulence and being chemically inert, it provides a three-dimensional crucible which permits the reagents to diffuse at a desirable controlled rate. Its softness and uniform nature of constraining forces that it exerts upon the growing crystals encourages orderly growth.

The growth of single crystals in gel at an ambient temperature, which are sparingly soluble in water, is a fascinating alternative to the techniques involving high temperature and expensive equipments. During the last few years, successful application of gel growth technique has been demonstrated by the preparation of single crystals of alkaline earth metal iodate. The gel growth technique appeared quite attractive for growing crystals of such compounds on account of its unique advantages in terms of crystals produced and the simplicity of process.

In the present work, crystals of mercuric iodate were grown by gel technique using diffusion method. Optimum growth conditions for crystals were determined. Optimum conditions were established by varying various parameters.

\section{Materials and Method}

Test tubes were used as crystallizing vessels. The silica gel was used as a growth media. Gel was prepared from aqueous solution of sodium meta silicate. The gel was acidified by acetic acid. The chemicals used for growth of single crystals of mercuric iodate were $\mathrm{CH}_{3} \mathrm{COOH} ; \mathrm{Na}_{2} \mathrm{SiO}_{3} .5 \mathrm{H}_{2} \mathrm{O} ; \mathrm{KIO}_{3}, \mathrm{NaIO}_{3} ; \mathrm{Hg}\left(\mathrm{NO}_{3}\right)_{2}$. All chemicals were of AR grade.

A series of experiments using different $\mathrm{pH}$ values for the gel and the different concentrations for reactants were carried out. Different molar masses were tried to determine the optimum growth conditions. Out of the two reactants, $\mathrm{Hg}\left(\mathrm{NO}_{3}\right)_{2}$ was incorporated in gel and $\mathrm{KIO}_{3}$ was used as the supernatant over the set gel. These experiments yield spherulitic crystals of $\mathrm{Hg}\left(\mathrm{IO}_{3}\right)_{2}$ of few $\mathrm{mm}$ size. Experiments were also carried out by interchanging the position of reactants. These experiments do not yield any crystals at all. $\mathrm{Hg}\left(\mathrm{NO}_{3}\right)_{2}$ having different concentrations was incorporated into the gel. This solution was then transferred to borosil glass tube of diameter $2.5 \mathrm{~cm}$ and $25 \mathrm{~cm}$ in height. The mouth of the tube was covered by cotton plug. After setting of the gel, it was left for aging for different periods of time. Another reactant having different concentrations was then added as supernatant over the set gel. Experiments were carried out by changing different concentrations of the reactants.

The chemical reaction inside the gel can be expressed as

$\mathrm{X}\left(\mathrm{NO}_{3}\right)_{2}+2 \mathrm{YIO}_{3} \rightarrow \mathrm{X}\left(\mathrm{IO}_{3}\right)_{2}+2 \mathrm{YNO}_{3}$

Where $\mathrm{X}=\mathrm{Hg}$ and $\mathrm{Y}=\mathrm{K}$ or $\mathrm{Na}$

\section{Results And Discussions}

Spherulite shaped crystals of 2 to $6 \mathrm{~mm}$ size were obtained. Study of kinetics of growth parameters reveals some interesting information. Spherulite shape of crystals is reported and has been explained previously. The shapes may be explained via a model of sheet of paper crumpled in to folds, the edges being compressed towards the centre. The result of this is a rounded crystal, but at first the basal planes (the plane of the sheet of the paper) are bent at random. These serves as the base for two-dimensional nuclei, which grow and fill up the recesses on the outer surface, thus giving crystal a spherical shape.

Higher density gel sets more rapidly. It decreases nucleation density. Increased density reduces diffusivity of ions which in turn reduces growth rate. Lower density gel takes long time to set and can be easily 
fractured. Increase in aging of gel reduces number of nucleation centres and growth rate. The reason may be the formation of additional cross-linkages between siloxane chains with increasing gel age, resulting in a gradually reducing cell size. This, in turn, reduces nucleation centres, since many nuclei find themselves in cells of very small size, where further growth is not possible. Insufficient gel aging leads to the formation of fragile gel and often breaks at the time of addition of supernatant. The effect of $\mathrm{pH}$ on growth rate was studied by changing $\mathrm{pH}$ without a change of gel composition and concentration of reactants. With $\mathrm{pH}$ values less than 4 , gel takes longer time to set and is unstable. There is no considerable effect on the quality of crystal. Higher $\mathrm{pH}$ value gel sets early, becomes turbid, and the size of crystal becomes smaller. As the $\mathrm{pH}$ increases, the gel structure changes from distinctly box-like network to a structure of loosely bound platelets, which appear to lack cross-linkages and the cellular nature becomes less distinct.

Less concentration of reactants does not yield any crystals at all. High concentration yields crystals of smaller size with increased nucleation centres. Reported concentration of reactants when used yields smaller spherulites near the gel interface with more number of nuclei. This may be due to high diffusion gradient near the gel interface. As the distance from the gel interface increases, number of nuclei reduces and size of spherulite increases due to smaller concentration gradient. Slow diffusion should lead to better nuclei, which because of their higher energy content should be less likely to reach their critical size.

\section{UV-VIS Specrophotometrry:}

Optical property of Mercuric iodate crystals can be studied using UV-VIS spectrophotometer. A fine powdered form of crystals was used as sample. The reflection and absorption spectra of Mercuric iodate crystals have been recorded over the wavelength range 200 to $700 \mathrm{~nm}$ using a UV-2450 spectrophotometer of SHIMADZU Scientific instruments at the room temperature. The experiment was carried out in the research laboratory of the physics department at pratap college, Amalner. With the help of this, absorption and reflection spectra are directly obtained through the computer using OOI base 32 software.

The absorption and reflection spectra of Mercuric iodate crystals recorded by UV-2450 spectrophotometer are as shown in the figures 1(a) and 1(b) respectively.

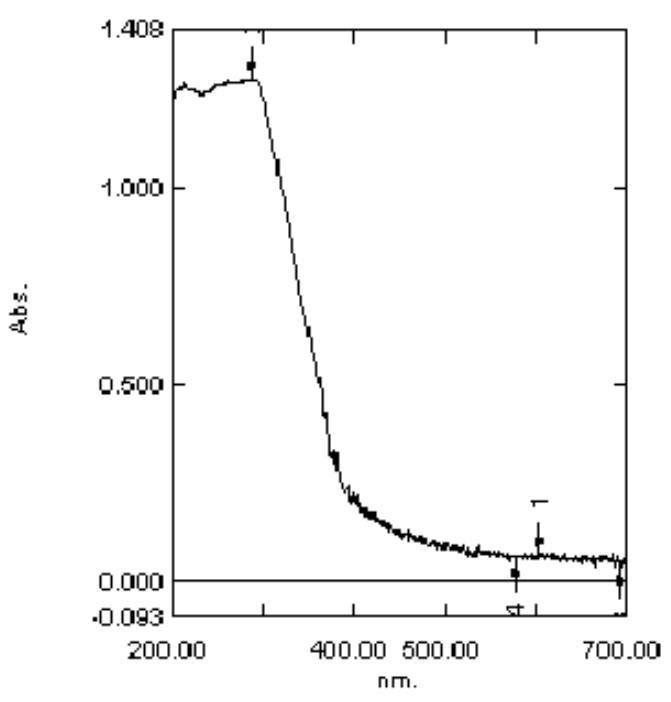

Figure.1(a) Absorption spectra

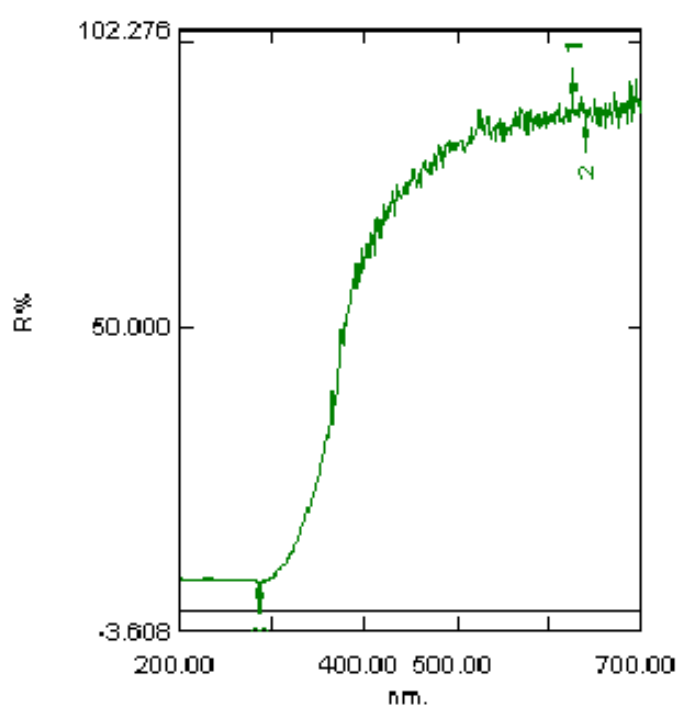

Figure.1(b) Reflection spectra

From the fig.1(a), it is observed that the absorption coefficient is high at lower wavelength and decreases sharply below a certain wavelength for Mercuric iodate crystals.

The excel data recorded by UV-2450 spectrophotometer is as shown in the table 1 . 


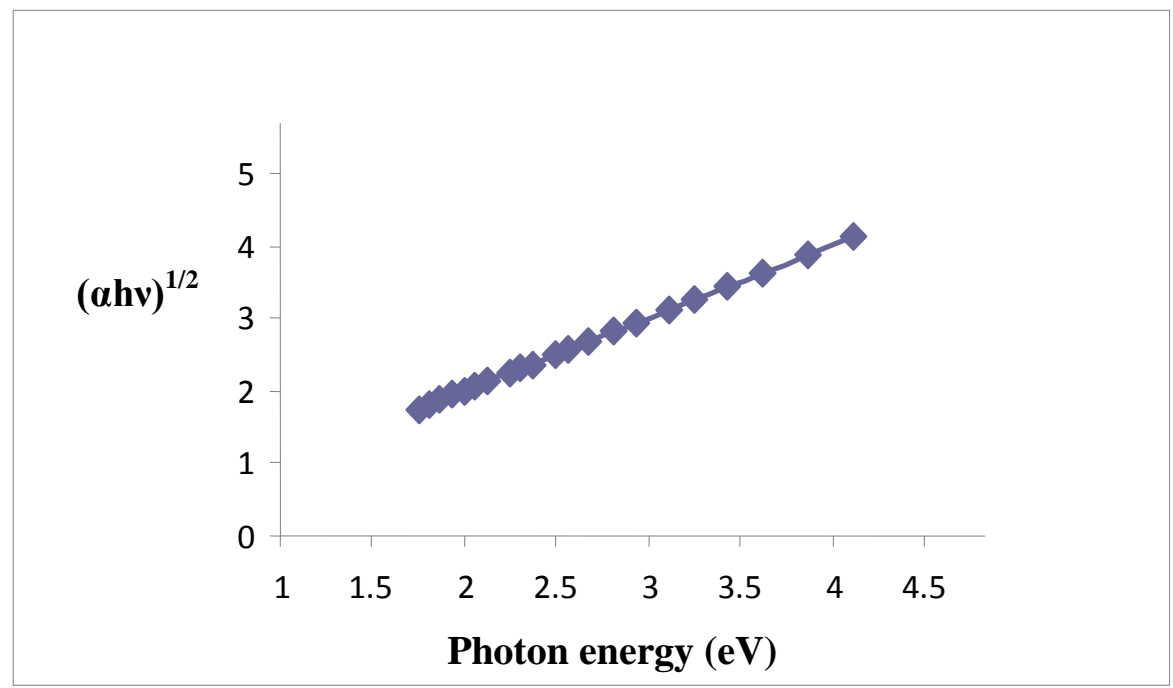

Figure 2(a) The graph of (hv) against (ahv) ${ }^{1 / 2}$

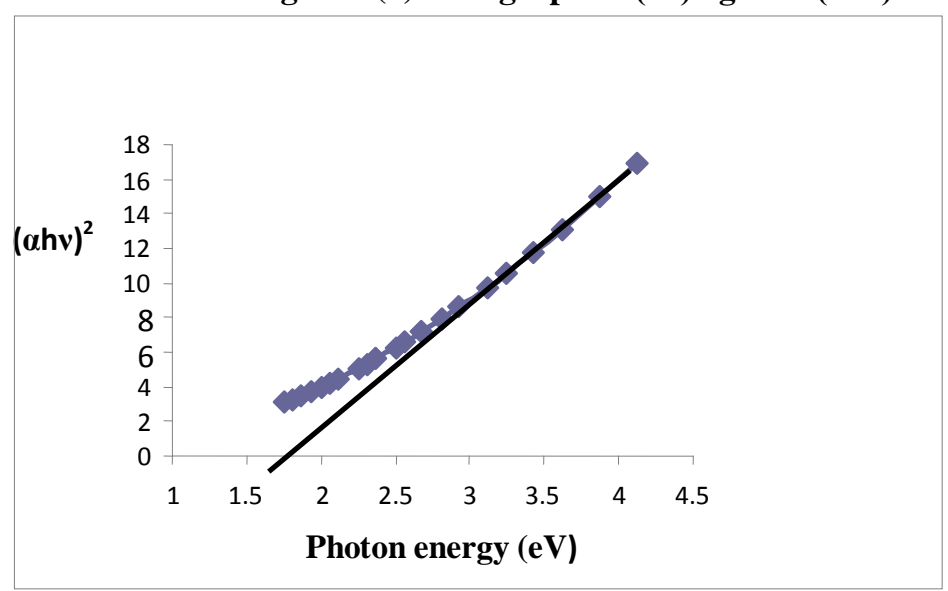

Figure 2(b) The graph of (hv) against (ahv) ${ }^{2}$

Table 1 : Excel data of mercuric iodate crystals

\begin{tabular}{|c|c|}
\hline$\lambda(\mathbf{n m})$ & Absorption coefficient $(\boldsymbol{\alpha})$ \\
\hline 300 & 1.226 \\
\hline 320 & 0.993 \\
\hline 340 & 0.736 \\
\hline 360 & 0.508 \\
\hline 380 & 0.325 \\
\hline 400 & 0.206 \\
\hline 420 & 0.17 \\
\hline 440 & 0.139 \\
\hline 460 & 0.12 \\
\hline 480 & 0.086 \\
\hline 500 & 0.08 \\
\hline 520 & 0.089 \\
\hline 540 & 0.07 \\
\hline 560 & 0.071 \\
\hline 580 & 0.063 \\
\hline 600 & 0.055 \\
\hline 620 & 0.068 \\
\hline 640 & 0.052 \\
\hline 660 & 0.052 \\
\hline 680 & 0.051 \\
\hline 700 & 0.057 \\
\hline
\end{tabular}


The band gap energy is determined from absorption spectra with the help of Tauc relation using the recorded excel data. Using this Tauc relation, a graph is plotted between the square root of ( $\alpha \mathrm{h} v)$ and hv (Photon energy) as shown in the figure 2(a) as well as between the square of $(\alpha h v)$ and $h v$ (Photon energy) as shown in the figure 2(b).

The extrapolation of straight line to $(\alpha \mathrm{h} v)^{2}$ axis in figure 2(b) gives the value of Optical band gap energy. The Optical energy band gap of the Mercuric iodate crystals is found to be $1.56 \mathrm{eV}$. It is in agreement with the value reported elsewhere.

From the above studies we observe that

\section{Conclusions}

(I) Gel growth technique is suitable for growing the crystals of Mercuric iodate.

(II) Single diffusion method is convenient for the growth of the Mercuric iodate crystals.

(III) Mercuric iodate crystals of appreciable size can be grown by single diffusion gel technique.

(IV) Gel growth technique is suitable for growing the crystals of mercuric iodate.

(V) The optical band gap energy of the grown crystals is found to be match very well with the reported elsewhere.

\section{Acknowledgements}

The authors are greatful to prof. Dr L A Patil, Principal and Head of the Department of physics, Pratap College, Amalner, for providing laboratory facilities. We are thankful to the staff and research scholar students of physics department of Pratap college, Amalner for providing the facility of UV-VIS spectrophotometer to carry out Optial property.

\section{References}

[1]. Joshi M S and Trivedi S G, Indian J. pure and App. Phys. 21435 (1983)

[2]. Shitole S J and Saraf K B , Bulletin of Material Science 5461 (2001)

[3]. Sangwal K and Patel A R, J. Crystal growth 23282 (1974)

[4]. Mitra, S. Fundamentals of Optical, Spectroscopic and X-ray Mineralogy, Wiley Eastern Limited (1989).

[5]. Little, L.H. Infrared Spectra of Adsorbed Species, Academic Press, London (1967).

[6]. Henisch, H. Crystal growth in gels, Pennsylvania state university press, University park, PA (1970).

[7]. Garud S L and Saraf K B , Bulletin of Material Science 4639 (2008)

[8]. Patil A B and Saraf K B , Asian Journal Of Chemical And Environmental Research (AJCER) (2011) 